01

\title{
Нелинейное уравнение Ланжевена для системы кулоновских частиц
}

\author{
(ㄱ Д.А. Жидков \\ ГНЦ РФ Институт теоретической и экспериментальной ффизики, \\ 117218 Москва, Россия \\ e-mail: dmtr7114@mail.ru
}

(Поступило в Редакцию 12 октября 2016 г. В окончательной редакции 21 октября 2017 г.)

Выведено и исследовано нелинейное уравнение Ланжевена для системы кулоновских частиц со случайными процессами, являющимися функционалами функции распределения этих частиц по скоростям. Прямыми численными решениями показано, что уравнение корректно описывает столкновительную релаксацию подобной системы даже в случае аномального отклонения начального распределения частиц по скоростям от равновесного. Уравнение удобно для использования в методах Монте-Карло и в методах „частицы-в-ячейке“.

DOI: 10.21883/JTF.2018.07.46160.2059

\section{Введение}

Столкновительная релаксация систем кулоновских частиц продолжает оставаться актуальной задачей ряда направлений ускорительной физики и физики плазмы. Долгое время единственным подходом к исследованию этой задачи было прямое решение уравнения Фокера-Планка (ФП) с интегралом столкновения Ландау [1]. Несмотря на существование хорошо отработанной методики для явного решения уравнения ФП [2-5], в последние годы активно разрабатываются подходы к моделированию кинетических свойств систем заряженных частиц, таких как метод Монте-Карло, метод „частицы-в-ячейке“ [6-11] и др. Причина заключается в том, что в отличие от технически сложного решения многомерного уравнения ФП, такие подходы позволяют проводить самосогласованное моделирование в переменных электромагнитных полях, особенно если эти поля ведут себя стохастически, что является обычным условием, например, для вещества с высокой плотностью энергии. Еще одним примером может служить плазма, создаваемая коротким интенсивным импульсом лазера, в котором важны и эффекты столкновений, и их отсутствие. Так, в явном моделировании уравнения ФП приходится принимать некоторую аналитическую модель механизма поглощения лазерного излучения [12], в то время как методы частиц позволяют это проводить самосогласованно.

Первая модель, призванная описывать столкновительную релаксацию систем заряженных частиц, была предложена Такезуке и Абе (ТА) [13]. Согласно этой модели, частицы объединяются в пары и упруго рассеиваются за каждый временной шаг. Однако не существует логического метода отделения температурной и динамической частей (средней скорости) в функции распределения при столкновении одинаковых частиц $[14,15]$. Так, например, вполне возможно рассеяние двух протонов в пучке с образованием частиц существенно разной энергии. Подобное разделение становится возможным при известной температуре $T$ и средней локальной скорости частиц $u$ потока частиц, т. е. определенной информации о функции распределения частиц по скоростям. Метод ТА также не применим для расчета эффекта поляризации плазмы в столкновениях частиц [1].

Метод уравнения Ланжевена для моделирования методом частиц менее известен. Долгое время существовало убеждение, что этот метод может быть использован только для расчета движения частиц в равновесном термостате с фиксированной температурой [16]. На примере кулоновских столкновений, однако, можно показать, что данный метод также может быть применен для расчета релаксации сильно неравновесных систем.

Метод уравнения Ланжевена основан на широко известной теореме, утверждающей эквивалентность распределения частиц по скоростям следующим из решения уравнением Ланжевена с распределением, являющимся решением уравнения ФП. А именно статистическое распределение скоростей, полученное из решения уравнения Ланжевена для системы с бесконечным числом частиц, должно полностью совпадать с решением уравнения ФП. Более того, эргодичность систем заряженных частиц позволяет подходить к решению уравнения ФП путем моделирования конечного числа частиц стохастической динамики.

Уравнение Ланжевена для учета кулоновских столкновений было предложено в работе [17]. Разнообразные подходы для уравнения Ланжевена также обсуждались в работах [18-22]. Тем не менее согласованность подхода к явному решению уравнения ФП была не ясна. В работе [21] была получена скорректированная форма уравнения Ланжевена, которая с использованием приближения Греда [1] для функции распределения частиц была применена для моделирования процесса убегания электронов в однородном электрическом поле. Было показано хорошее согласие с результатами прямого моделирования ФП потока убегания электронов от напряженности электрического поля и заряда ионов. Более того, в отличие от моделирования ФП, в котором используются только максвелловские столкновения, моделирование [21] обеспечило спицеровскую проводи- 
мость при малых электрических полях. В этом приближении уравнение успешно применялось для полного кинетического моделирования сверхплотной плазмы, облученной короткоимпульсным лазером [22]. Однако все расчеты проводились в условиях, когда функция распределения частиц по скоростям не сильно отличается от равновесной.

В настоящей работе выводится и исследуется полностью нелинейное уравнение Ланжевена для системы нерелятивистских кулоновских частиц одинаковой массы. Такая система выбрана из-за простоты и ясности подхода к выводу уравнения Ланжевена, хотя основные результаты могут быть применены и к релятивистским системам, и к процессам, в которых силы определяются отличными от столкновений причинами. Исследование применимости выведенного уравнения к моделированию реальных процессов проводится на примере релаксации системы кулоновских частиц с начальным распределением по скоростям, которое существенно отличается от равновесного распределения. Так рассчитывается временная релаксация системы с распределением, дающимся изначально изотропной функцией Хевисайда $f(v)=A \eta\left(1-v / v_{0}\right)$ и изотропной $\delta$-функцией, $f(v)=A \delta\left(1-v / v_{0}\right)$, где $v_{0}-$ средняя тепловая скорость частиц.

\section{Нелинейное уравнение Ланжевена}

Уравнение Ланжевена должно выводиться на основе его тесной связи с уравнением ФП. Как известно, уравнение ФП для функции распределения $f_{\alpha}$ имеет следующий вид [1]:

$$
\begin{aligned}
\frac{\partial f_{\alpha}}{\partial t}+ & \mathbf{v} \frac{\partial f_{\alpha}}{\partial r}+\frac{q_{\alpha}}{M_{\alpha}}\left[\mathbf{E}+\frac{\mathbf{v}}{c} \mathbf{H}\right] \frac{\partial f_{\alpha}}{\partial \mathbf{v}}=-\sum_{\beta} \frac{\partial}{\partial v_{i}} \\
& \times\left[A_{i \alpha \beta} f_{\alpha}-\frac{1}{2} \frac{\partial}{\partial v_{j}}\left(B_{i j \alpha \beta} f_{\alpha}\right)\right] .
\end{aligned}
$$

где $q_{\alpha}, M_{\alpha}$ - заряд и масса частицы $\alpha$. Матрицы в интеграле столкновения записываются в виде [23]

$$
A_{i \alpha \beta}(\mathbf{v})=\lim _{\Delta t \rightarrow 0} \frac{1}{\Delta t}\left\langle\Delta v_{i \alpha \beta}\right\rangle=N_{\beta} \int f_{\beta}\left(\mathbf{v}_{\beta}\right) w_{i}^{\alpha \beta} d \mathbf{v}_{\beta},
$$

$$
B_{i j \alpha \beta}(\mathbf{v})=\lim _{\Delta t \rightarrow 0} \frac{1}{\Delta t}\left\langle\Delta v_{i \alpha \beta} \Delta v_{j \alpha \beta}\right\rangle=N_{\beta} \int f_{\beta}\left(\mathbf{v}_{\beta}\right) w_{i j}^{\alpha \beta} d \mathbf{v}_{\beta},
$$

где $\Delta v_{i}$ - изменение скорости частицы вследствие столкновения в промежуток времени $\Delta t,\langle\ldots\rangle$ означает усреднение по ансамблю частиц. Для бинарных столкновений корреляционные функции в уравнении (2a) определяются сечениями рассеяния

$$
w_{i}^{\alpha \beta}=-\left(\frac{\mu_{\alpha \beta}}{M_{\alpha}}\right)\left(v_{i \alpha}-v_{i \beta}\right)\left|\mathbf{v}_{\alpha}-\mathbf{v}_{\beta}\right| \sigma_{1}\left(\left|\mathbf{v}_{\alpha}-\mathbf{v}_{\beta}\right|\right),
$$

$$
\begin{aligned}
w_{i j}^{\alpha \beta}= & \left(\frac{\mu_{\alpha \beta}}{M_{\alpha}}\right)^{2}\left|\mathbf{v}_{\alpha}-\mathbf{v}_{\beta}\right|^{3}\left[\delta_{i j} \frac{\sigma_{2}}{2}+\frac{\left(v_{i \alpha}-v_{i \beta}\right)\left(v_{j \alpha}-v_{j \beta}\right)}{\left|\mathbf{v}_{\alpha}-\mathbf{v}_{\beta}\right|^{2}}\right. \\
& \left.\times\left(2 \sigma_{1}-\frac{3}{2} \sigma_{2}\right)\right], \\
\sigma_{1}= & \int(1-\cos \theta) d \sigma, \quad \sigma_{2}=\int\left(1-\cos ^{2} \theta\right) d \sigma .
\end{aligned}
$$

Дифференциальное сечение $d \sigma$ зависит от процесса столкновения, приведенная масса $\mu_{\alpha \beta}=M_{\alpha} M_{\beta}$ / $\left(M_{\alpha}+M_{\beta}\right)$. Для кулоновских столкновений

$$
\sigma_{1}\left(\left|\mathbf{v}-\mathbf{v}^{\prime}\right|\right)=\frac{1}{2} \sigma_{2}\left(\left|\mathbf{v}-\mathbf{v}^{\prime}\right|\right)=\frac{4 \pi e_{\alpha}^{2} e_{\beta}^{2} \Lambda}{\mu_{\alpha \beta}^{2}\left|\mathbf{v}-\mathbf{v}^{\prime}\right|^{4}},
$$

где $e_{\alpha}, e_{\beta}$ - заряды частиц, $\Lambda$ - кулоновский логарифм, а коэффициенты $A$ и $B$ выражаются через потенциалы Розенблюта [23],

$$
\begin{gathered}
A_{i \alpha \beta}(\mathbf{v})=\frac{4 \pi e_{\alpha}^{2} e_{\beta}^{2} \Lambda}{M_{\alpha} \mu_{\alpha \beta}} N_{\beta} \frac{\partial}{\partial v_{\alpha i}} \int \frac{f_{\beta}\left(\mathbf{v}_{\beta}\right)}{\left|\mathbf{v}_{\alpha}-\mathbf{v}_{\beta}\right|} d \mathbf{v}_{\beta}, \\
B_{i j \alpha \beta}(\mathbf{v})=\frac{4 \pi e_{\alpha}^{2} e_{\beta}^{2} \Lambda}{M_{\alpha}^{2}} N_{\beta} \frac{\partial^{2}}{\partial v_{\alpha i} \partial v_{\alpha j}} \int f_{\beta}\left(\mathbf{v}_{\beta}\right)\left|\mathbf{v}_{\alpha}-\mathbf{v}_{\beta}\right| d \mathbf{v}_{\beta} .
\end{gathered}
$$

Выделяя векторную часть, потенциалы Розенблюта можно представить в следующем виде:

$$
\mathbf{A}_{\alpha \beta}(\mathbf{v})=F(v) \frac{\mathbf{v}}{v^{3}}, \quad B_{i j}(\mathbf{v})=G(v) \delta_{i j}-F(v) \frac{v_{i} v_{j}}{v^{2}},
$$

где скалярные функции $F(v)>G(v)>0$ зависят от всех компонент скорости $v=\left(v_{x}, v_{y}, v_{z}\right)$ как

$$
\begin{aligned}
& F(\mathbf{v})=\frac{4 \pi e_{\alpha}^{2} e_{\beta}^{2} \Lambda}{M_{\alpha}^{2}} N_{\beta} v^{2} \int \frac{f_{\beta}\left(\mathbf{v}_{\beta}\right) d \mathbf{v}_{\beta}}{\left|\mathbf{v}-\mathbf{v}_{\beta}\right|^{3}}, \\
& G(\mathbf{v})=\frac{4 \pi e_{\alpha}^{2} e_{\beta}^{2} \Lambda}{M_{\alpha}^{2}} N_{\beta} v^{2} \int \frac{f_{\beta}\left(\mathbf{v}_{\beta}\right) d \mathbf{v}_{\beta}}{\left|\mathbf{v}-\mathbf{v}_{\beta}\right|} .
\end{aligned}
$$

Вычисление коэффициентов требует трехмерного интегрирования в пространстве скоростей, которое является трудоемкой процедурой. В случае же изотропного распределения все расчеты сводятся к трем одномерным интегралам. Первый

$$
I_{p}(v)=\int_{0}^{v} f(z) z^{2} d z, \quad I_{p}(\infty)=1
$$

— число частиц в сфере радиуса $v$, второй

$$
I_{E}(v)=\int_{0}^{v} f(z) z^{4} d z
$$

- нормированная полная кинетическая энергия частиц со скоростями $z<v$, третий

$$
I_{\sigma}(v)=\int_{v}^{\infty} f(z) z d z \infty\left\langle v^{2} \sigma(v) v N\right\rangle
$$


- вероятность электрона со скоростью $z>v$ потерять часть своей энергии при столкновении.

Нерелятивистское уравнение Ланжевена для движения заряженных частиц в произвольном поле с учетом столкновений может быть записано в виде

$$
M_{\alpha} \frac{d v_{\alpha}}{d t}=q_{\alpha}\left[\mathbf{E}\left(\mathbf{r}_{\alpha}\right)+\frac{v_{\alpha}}{c} \mathbf{H}\left(\mathbf{r}_{\alpha}\right)\right]+M_{\alpha} \mathbf{P}\left(\mathbf{v}_{\alpha}\right),
$$

где $v_{\alpha}-$ скорость, $\mathbf{r}_{\alpha}-$ координата, $q_{\alpha}, M_{\alpha}-$ заряд и масса частицы сорта $\alpha, \mathbf{E}$ и $\mathbf{H}$ - электрическое и магнитное поля, $\mathbf{P}\left(\mathbf{v}_{\alpha}\right)$ - стохастическая сила, определяемая столкновениями. И в общем виде ее можно записать как $\mathbf{P}\left(\mathbf{v}_{\alpha}\right)=\mathbf{f}_{\alpha}+\mathbf{d}_{\sigma} \boldsymbol{\xi}$, где $\mathbf{f}_{\alpha}-$ торможение, $\mathbf{d}_{\alpha}$ - матрица, зависящая от скорости и функции распределения частиц, $\xi$ описывает нормальный случайный процесс с характеристиками

$$
\begin{gathered}
\left\langle\xi_{i}(t)\right\rangle=0 ; \quad\left\langle\xi_{i}(t) \xi_{k}(t+\Delta t)\right\rangle=\delta_{i k} \delta(\Delta t), \\
\left\langle\xi_{i}(t) \eta_{k}(t+\Delta t)\right\rangle=0, \quad \int_{i}^{t+\Delta t} \xi_{k} d t^{\prime}=p_{k} \sqrt{\Delta t},
\end{gathered}
$$

где $p_{k}$ - число из набора случайных чисел с нормальным распределением.

Для соответствия уравнению ФП уравнение (4) должно обеспечить функции корреляции скоростей (2a). Это означает, что при $\mathbf{E}=0, \mathbf{H}=0$ должны выполняться равенства

$$
\begin{gathered}
\lim \frac{1}{\Delta t}\left\langle\int_{t}^{t+\Delta t} P_{i}(v) d t\right\rangle=A_{i}(v), \\
\lim \frac{1}{\Delta t}\left\langle\int_{t}^{t+\Delta t} P_{i}(v) d t \int_{t}^{t+\Delta t} P_{i}(v) d t^{\prime}\right\rangle=B_{i j}(v) .
\end{gathered}
$$

Очевидно, что матрица $d$ пропорциональна $\left(B_{i j}\right)^{1 / 2}$. Однако матрица $B_{i j}(3 \mathrm{~b})$ имеет четыре точных корня

$$
\begin{aligned}
\left(B_{i j}(v)\right)^{1 / 2} & = \pm\left[\sqrt{G(v)} \delta_{i j}\right. \\
& \left.-\left(\sqrt{G(v)} \pm \sqrt{G(v)-F(v)} \frac{v_{i} v_{j}}{v^{2}}\right)\right]
\end{aligned}
$$

где $F$ и $G$ определяются уравнением (3c) и уравнением (3d). Если знаком корня можно пренебречь из-за вырождения, то два знака внутри корня матрицы приводят к разным корням. Поэтому стохастическая сила в уравнении Ланжевена в представлении гауссовских процессов должна иметь вид $\mathbf{P}\left(v_{\alpha}\right)=\mathbf{f}_{\alpha}+\hat{\mathbf{d}}_{\alpha} \xi+\hat{\mathbf{g}}_{\alpha} \eta$, где два независимых процесса и

$$
f_{i}=A_{i}, \quad d_{i k}=\frac{1}{\sqrt{2}}\left(B_{i k+}\right)^{1 / 2}, \quad g_{i k}=\frac{1}{\sqrt{2}}\left(B_{i k-}\right)^{1 / 2} .
$$

\section{Релаксация изотропного распределения}

Для проверки применимости метода уравнения Ланжевена процесс релаксации первоначально изотропного, сильно неравновесного распределения системы одинаковых частиц по скоростям к максвелловскому распределению удобно рассматривать в отсутствие внешних полей. Окончательный вид уравнения Ланжевена может быть получен аналитически для изотропного распределения одинаковых частиц с помощью формул (4). Уравнение Ланжевена для столкновений одинаковых частиц с изотропным распределением в системе отсчета, движущейся вместе с частицами, имеет следующий вид:

$$
\begin{aligned}
& \frac{d \mathbf{v}}{d t}=-4_{\gamma} \frac{\mathbf{v}}{v}\left(\frac{I_{p}(u)}{2 u^{2}}\right) \\
& +\sqrt{\frac{\gamma}{u}}\left\{\sqrt{I_{p}(u)+\frac{2}{3} u I_{\sigma}(u)-\frac{1}{3 u^{2}} I_{E}(u)}\right. \\
& \times\left[\xi+\eta-\frac{\mathbf{v}}{v^{2}}(\mathbf{v}, \xi+\boldsymbol{\eta})\right]+\sqrt{\frac{2}{3} u I_{\sigma}(u)+\frac{2}{3 u^{2}} I_{E}(u)} \\
& \left.\times \frac{\mathbf{v}}{v^{2}}(\mathbf{v}, \xi-\boldsymbol{\eta})\right\}, \\
& \sum \mathbf{v}=0, \quad v_{0}=\sqrt{\frac{2}{3 N} \sum v^{2}}, \quad \gamma=4 \pi \Lambda\left(\frac{e_{\alpha}^{2}}{M_{\alpha}}\right)^{2} \frac{N_{\alpha}}{v_{0}^{3}}
\end{aligned}
$$

где $\quad u=v / v_{0},(\mathbf{v}, \boldsymbol{\xi}+\boldsymbol{\eta})=(\mathbf{v}, \boldsymbol{\xi})+(\mathbf{v}, \boldsymbol{\eta})$ - скалярное умножение, $(\mathbf{v}, \boldsymbol{\xi})=\left(v_{1} \xi_{1}+v_{2} \xi_{2}+v_{3} \xi_{3}\right)$. Интегралы $I_{p}(u), I_{E}(u), I_{\sigma}(u)$ определены в предыдущем разделе. Они зависят от функции распределения частиц. Слагаемые в квадратных корнях всегда положительны. В соответствии с уравнением (7) из-за столкновений происходит изменение энергии частицы

$$
\begin{aligned}
\frac{d \varepsilon}{d t}=\mathbf{v} \frac{d \mathbf{v}}{d t}= & -4 \gamma v\left(\frac{I_{p}(u)}{2 u^{2}}\right)+\sqrt{\frac{2 \gamma}{3 u}\left[u I_{\sigma}(u)+\frac{1}{u^{2}} I_{E}(u)\right]} \\
& \times(\mathbf{v}, \boldsymbol{\xi}-\boldsymbol{\eta}) .
\end{aligned}
$$

Это перераспределение энергии обеспечивает эргодичность системы, даже если нет внешней силы, полная энергия сохраняется точно для бесконечного числа частиц

$$
\left.\frac{1}{N} \sum_{i=1}^{N} \frac{d \varepsilon_{i}}{d t}\right|_{N \rightarrow \infty}=0
$$

Как показано ниже прямым численным расчетом, усредненная по времени полная энергия сохраняется даже для конечного, сравнительно низкого числа частиц.

Для решения уравнения (7) использовалась явная схема первого порядка точности с коррекцией энергии 

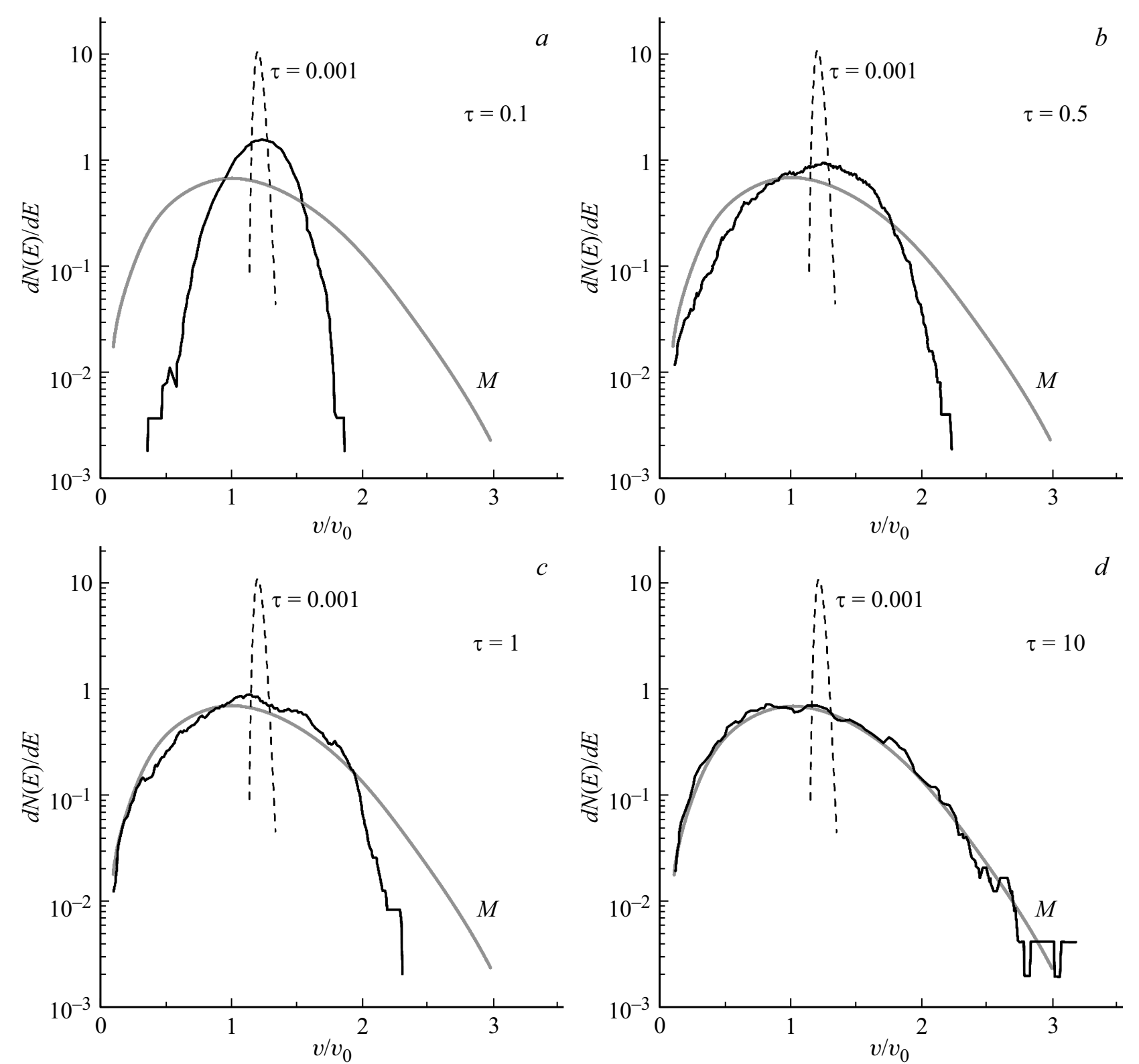

Рис. 1. Релаксация системы $N=1000$ одинаковых заряженных частиц с начальным распределением по скоростям (9а) согласно решению уравнения (8). $a-$ время равно 0.1 столкновительного, $b-0.5, c-1.0, d-10$ столкновительных времен. Кривая $M$ обозначает равновесное максвелловское распределение. Начальное распределение дано для $\tau=0.001$.

и импульса в следующем виде:

$$
\begin{aligned}
& \mathbf{v}_{k}^{+}=\mathbf{v}_{k}-4 \frac{\mathbf{v}_{k}}{v_{k}} \Delta t\left(\frac{I_{p}\left(u_{k}\right)}{2 u_{k}^{2}}\right) \\
& +\sqrt{\frac{\Delta t}{u_{k}}}\left\{\sqrt{I_{p}\left(u_{k}\right)+\frac{2}{3} u_{k} I_{\sigma}\left(u_{k}\right)-\frac{1}{3 u_{k}^{2}} I_{E}\left(u_{k}\right)}\right. \\
& \times\left[\mathbf{p}_{1 k}+\mathbf{p}_{2 k}-\frac{\mathbf{v}_{k}}{v_{k}^{2}}\left(\mathbf{v}_{k}, \mathbf{p}_{1 k}+\mathbf{p}_{2 k}\right)\right] \\
& \left.+\sqrt{\frac{2}{3} u_{k} I_{\sigma}\left(u_{k}\right)+\frac{2}{3 u_{k}^{2}} I_{E}\left(u_{k}\right)} \frac{\mathbf{v}_{k}}{v_{k}^{2}}\left(\mathbf{v}_{k}, \mathbf{p}_{1 k}-\mathbf{p}_{2 k}\right)\right\},
\end{aligned}
$$

где $k=1, \ldots, N-$ порядковый номер частицы, $v^{+}=v_{n+1}$, $v=v_{n}, u=v_{n} / v_{0 n}, \Delta \tau=\gamma \Delta t, \gamma$ - частота столкновений при $v=v_{0 n}, v_{0}=N^{-1} \sqrt{2 \sum v_{k}^{2} / 3}$. После расчета скорости может быть применена линейная поправка, $\hat{v}_{k}^{+}=\left(v_{k}^{+}-V_{n+1}\right) v_{0 n} / v_{0 n+1}$, где средняя скорость $V_{n+1}$ оценивается как $v_{n+1}=N^{-1} \sum v_{k}^{+}$. Расхождение $D=$ $=v_{0 n}^{2} / v_{0 n+1}^{2}-1$ определяет ошибку расчета.

Для решения системы (8) нужно сначала вычислить функцию распределения частиц в каждом шаге по времени, а затем вычислить интегралы $I_{\sigma}, I_{p}, I_{E}$. Для этого удобно начать с вычисления $I_{p}$ простой выборкой на равномерной сетке с шагом $\Delta v=\left(v_{\max } / v_{0}\right) / M$, где $v_{\max }$ и $M-$ максимальная скорость частиц и раз- 

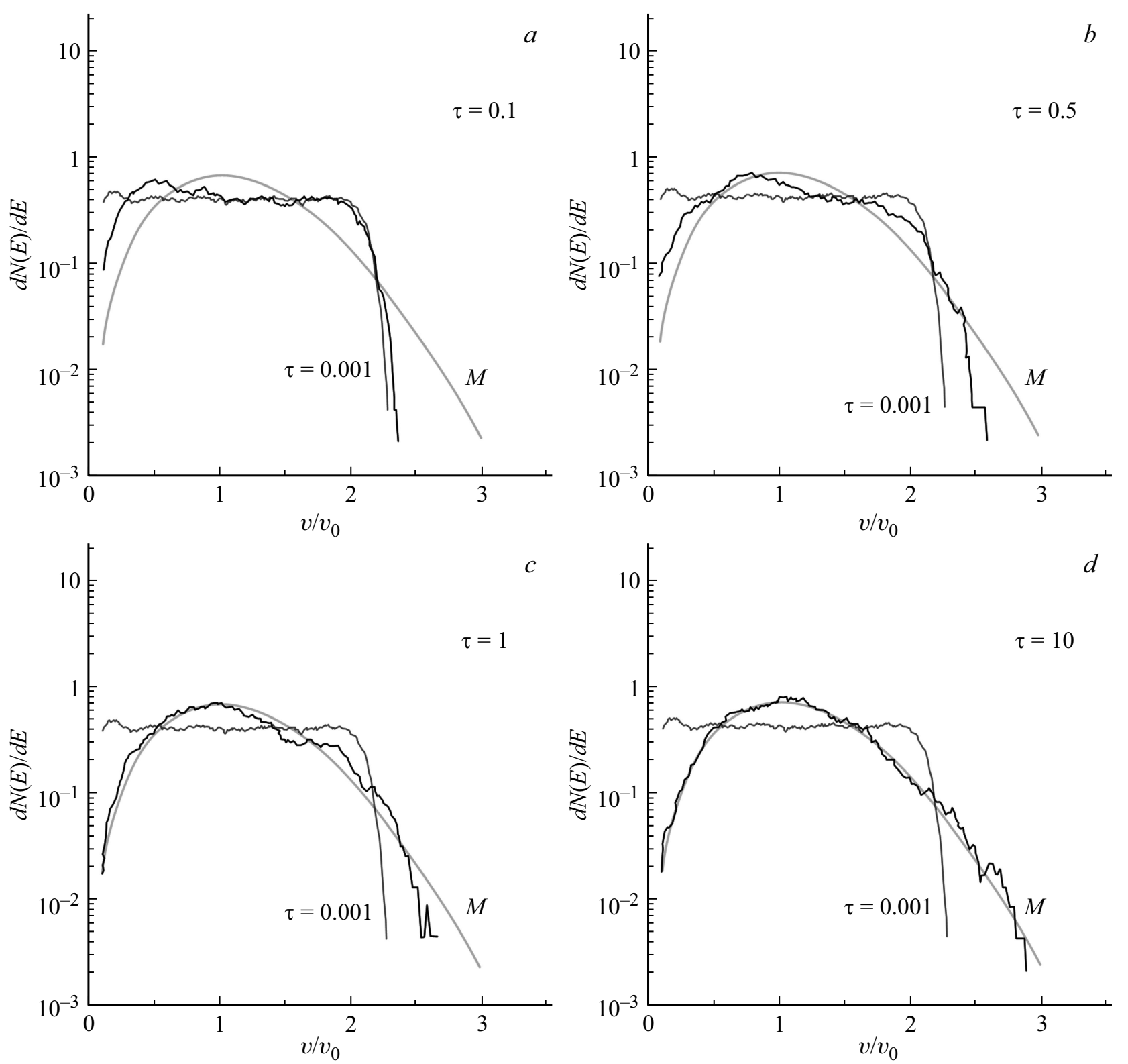

Рис. 2. Релаксация системы $N=1000$ одинаковых заряженных частиц с начальным распределением по скоростям (9b). $a-$ время равно 0.1 столкновительного, $b-0.5, c-1.0, d-10$ столкновительных времен. Кривая $M$ обозначает равновесное максвелловское распределение. Начальное распределение дано для $\tau=0.001$.

мер сетки, а затем вычислять функцию распределения $f(j)=\left[I_{p}(j+1)-I_{p}(j)\right] / 2 \Delta v v_{j}^{2}$. Расчет $I_{\sigma}(j)$ и $I_{E}(j)$ тривиальны.

Начальные распределения частиц по скоростям выбираются в виде

$$
\begin{gathered}
f^{(1)}(v)=2 \delta\left(\sqrt{3 / 2}-v / v_{0}\right) / 3 v_{0}^{3}, \\
f^{(2)}(v)=3(2 / 5)^{3 / 2} \eta\left(\sqrt{5 / 2}-v / v_{0}\right) / v_{0}^{3},
\end{gathered}
$$

где $\eta(x), \delta(x)$ являются стандартными функциями.

Временная эволюция функции распределения, изначально определяемая формулами (8a), (8b), представлена на рис. 1 и 2. Расчет производится для $N=1000$ и временного шага $\Delta \tau=10^{-3}$ для детального представления процесса релаксации. Для обоих распределений по скоростям как по дельта-функции, так и для распределения Хевисайда процесс релаксации устойчив. Функции распределения стремятся к своим равновесным значениям за времена, определяемые частотой столкновений. Время релаксации ядра распределения приблизительно равно времени столкновения, тогда как время релаксации распределения в области высоких энергий $v \sim 10 v_{0}$ к максвелловской, очевидно, происходит дольше и зависит от энергии частиц. Например, релаксация в скоростях $v=3 v_{0}$ завершается после 10 столкновений. Следуя работе [15], мы провели аналогичный расчет с 


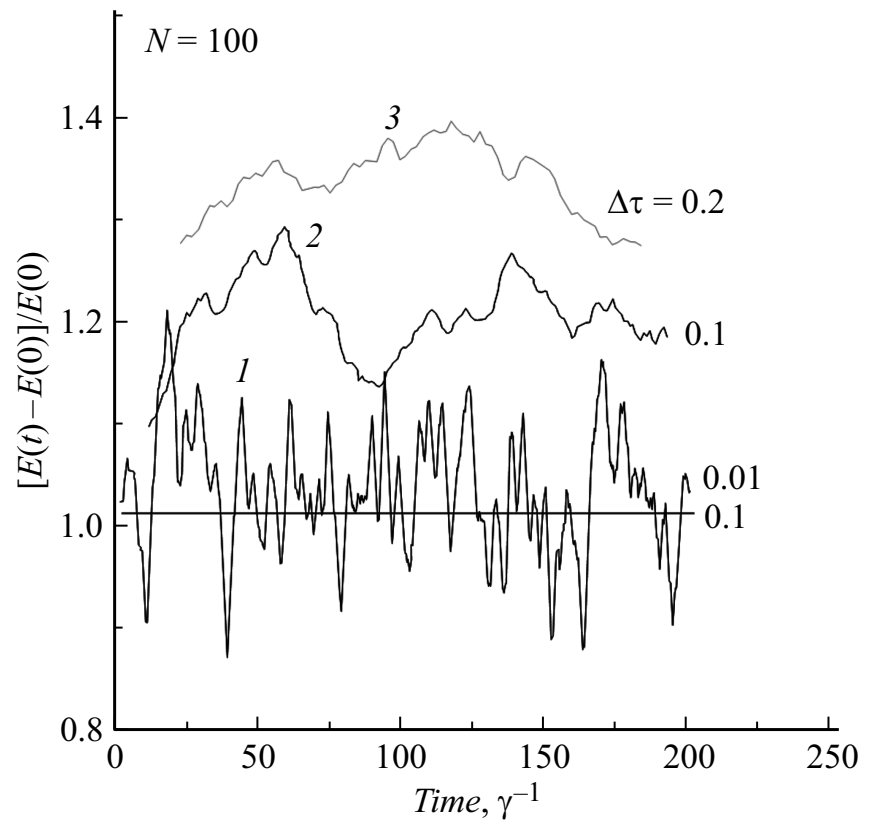

Pис. 3. Изменение полной энергии частиц без коррекции средней скорости для $\Delta t=0.2 \gamma^{-1}, \Delta t=0.1 \gamma^{-1}, \Delta t=0.01 \gamma^{-1}$ и (прямая линия) при коррекции и $\Delta t=0.1 \gamma^{-1}$.

использованием метода ТА. Для обеспечения релаксации мы должны были использовать $N=20000$ частиц.

На рис. 3 показано расхождение полной энергии частиц при расчете более 200 столкновений. Расчеты выполнены с использованием и без коррекции средней скорости с произвольным начальным распределением. В начале релаксации общее изменение энергии зависит от временного шага и числа частиц. Когда система частиц достигает равновесия, полная энергия сохраняется даже при $\Delta \tau=1$. Если производить коррекцию энергии импульса после каждого временного шага, максимальное изменение энергии системы $D$ будет меньше $10^{-3}$ от полной энергии при $N=100$ и $\Delta \tau=0.1$. Такие параметры подходят для моделирования реальной системы заряженных частиц, например для взаимодействия пучка со средой или для плотной плазмы.

\section{Заключение}

Релаксация системы одинаковых частиц (электронов или ионов), вызванных кулоновскими столкновениями, была рассчитана с помощью нелинейного уравнения Ланжевена. Рассматривалась система $N \sim 10^{2}-10^{3}$ частиц с первоначально изотропным распределением по скоростям, которое сильно отличалось от равновесного. Было показано, что даже явная численная схема первого порядка с коррекцией энергии и импульса обеспечивает эффективное численное решение релаксации для произвольной системы с изотропным распределением скоростей при от- носительно низком числе частиц и большим шагом по времени. Исследованы временные эволюции системы $N=1000$ частиц для распределений изотропных степенной функции и дельта-функции. Время релаксации находится в допустимом согласии со временем, следующим из прямого решения уравнения Фоккера-Планка.

Поскольку решение уравнений (5) с силой (7) полностью идентично решению уравнения (1), представленный метод может быть использован для любых распределений частиц, в том числе сильно анизотропных. Однако в общем расчете становится необходимым расширенное вычисление значений трехмерных интегралов в формулах (4). И хотя использование приближения Греда позволяет упростить расчет релаксации, в случае сильных искажений начальных распределений может требоваться решение нелинейного уравнения Ланжевена. Следует отметить один недостаток метода уравнения Ланжевена: в отличие от ФП моделирования моделирование методами частиц не дает корректную оценку пространственных производных таких физических величин, как температура, плотность и скорость потока.

\section{Список литературы}

[1] Силин В.П. Введение в кинетическую теорию газов. М.: Наука, 1971.

[2] Killeen J., Futch A.H. // J. Comput. Phys. 1968. Vol. 2. P. 236.

[3] Berezin Y.A., Khudnik V.N., Pekker M.S. // J. Comput. Phys. 1987. Vol. 69. P. 163.

[4] Empperlen E.M. // J. Comput. Phys. 1994. Vol. 111. P. 291.

[5] Buet C., Cordier S. // J. Comput. Phys. 1998. Vol. 145. P. 228.

[6] Turner M.M. // Phys. Plasmas. 2006. Vol. 13. P. 033506.

[7] Turner M.M. // Plasma Soucrce Sci. Technol. 2016. Vol. 25. P. 054007.

[8] Chen Z., Ye Q., Xia G. et al. // Phys. Plasmas. 2016. Vol. 20. P. 033502.

[9] David L.R. Collisional Particle In Cell Modelling Of The Propagation Of Fast Electrons In Solid Density Plasma. PhD Thesis, Imperial College. London, 2013.

[10] Бэдсел Ч., Ленгдон А. // Физика плазмы и численное моделирование / Пер. с англ. М.: Энергоатомиздат, 1989. $452 \mathrm{c}$.

[11] Langdon A.B. // Phys. Rev. Lett. 1980. Vol. 44. P. 575.

[12] Town R.P., Bell A.R., Rose S.J. // Phys. Rev. Lett. 1995. Vol. 74. P. 924.

[13] Takizuka T., Abe H. // J. Comput. Phys. 1977. Vol. 25. P. 205.

[14] Birdsall C.K. // IEEE Trans. Plasma Sci. 1991. Vol. 19. P. 65.

[15] Nanbu K. // Phys. Rev. 1997. Vol. E55. P. 4642.

[16] Van Kampen V.G. Stochastic Processes in Physics and Chemistry. North-Holland, Amsterdam, 1984.

[17] Ivanov M.F., Svets V.F. // Sov. Phys. Dokl. 1978. Vol. 23. P. 130.

[18] Zhidkov A. et al. // Laser Phys. 1994. Vol. 4. P. 1131.

[19] Cadjan M.G., Ivanov M.F. // Phys. Lett. A. 1997. Vol. 236. P. 227. 
[20] Cadjan M.G., Ivanov M.F. Proceedings of 16th Int. Conf. on the Numerical Plasma Simulation, US. 1998.

[21] Zhidkov A. // Phys. Plasmas. 1998. Vol. 5. P. 385.

[22] Zhidkov A., Sasaki A. // Phys. Rev. 1999. Vol. E57. P. 7085; Zhidkov A.G., Sasaki A., Tajima T. et al. // Phys. Rev. 1999. Vol. E60. P. 3273.

[23] Трубников Б.А. Столкновение частиц в полностью ионизованной плазме. В сб.: Вопросы теории плазмы. Т. 1. / Под ред. М.А. Леонтовича. М.: Энергоатомиздат, 1973. 